\title{
Limitations of Using Pediatric Respiratory Illness Readmissions to Compare Hospital Performance
}

\author{
Sunitha V. Kaiser, MD, MSc ${ }^{1 *}$, Regina Lam, BA1', Gabby B. Joseph, PhD1', Charles McCulloch, PhD', \\ Renee Y. Hsia, MD, MSc ${ }^{1,2}$, Michael D. Cabana, MD, MPH',2, Naomi S. Bardach, MD, MS 1,2
}

${ }^{1}$ University of California, San Francisco, California; ${ }^{2}$ Phillip R. Lee Institute for Health Policy Studies, San Francisco, California.

BACKGROUND: Adult hospital readmission rates can reliably identify meaningful variation in hospital performance; however, pediatric condition-specific readmission rates are limited by low patient volumes.

OBJECTIVE: To determine if a National Quality Forum (NOF)-endorsed measure for pediatric lower respiratory illness (LRI) 30-day readmission rates can meaningfully identify high- and low-performing hospitals.

DESIGN: Observational, retrospective cohort analysis. We applied the pediatric LRI measure and several variations to evaluate their ability to detect performance differences.

SETTING: Administrative claims from all hospital admissions in California (2012-2014).

PATIENTS: Children (age $<18$ years) with LRI (primary diagnosis: bronchiolitis, influenza, or pneumonia; or LRI as a secondary diagnosis with a primary diagnosis of respiratory failure, sepsis, bacteremia, or asthma).

MEASUREMENTS: Thirty-day hospital readmission rates and costs. Hierarchical regression models adjusted for age, gender, and chronic conditions were used.

RESULTS: Across all California hospitals admitting children $(n=239)$ using respiratory readmission rates, no outlier hospitals were identified with (1) the NQF-endorsed metric, (2) inclusion of primary asthma or secondary asthma exacerbation diagnoses, or (3) inclusion of 30-day emergency revisits. By including admissions for asthma, adding emergency revisits, and merging 3 years of data, we identified 9 outlier hospitals ( 2 high-performers, 7 low-performers). There was no association of hospital readmission rates with costs.

CONCLUSIONS: Using a nationally-endorsed quality measure of inpatient pediatric care, we were unable to identify meaningful variation in hospital performance without broadening the metric definition and merging multiple years of data. Utilizers of pediatric-quality measures should consider modifying metrics to better evaluate the quality of pediatric care at low-volume hospitals. Journal of Hospital Medicine 2018; 13:737-742. Published online first July 25, 2018. ( Society of Hospital Medicine espiratory illnesses are the leading causes of pediatric hospitalizations in the United States. ${ }^{1}$ The 30 -day hospital readmission rate for respiratory illnesses is being considered for implementation as a national hospital performance measure, as it may be an indicator of lower quality care (eg, poor hospital management of disease, inadequate patient/caretaker education prior to discharge). In adult populations, readmissions can be used to reliably identify variation in hospital performance and successfully drive efforts to improve the value of care. ${ }^{2,3}$ In contrast, there are persistent concerns about using pediatric readmissions to identify variation in hospital performance, largely due to lower patient volumes. ${ }^{4.7}$ To increase the value of pediatric hospital care, it

\footnotetext{
*Address for correspondence: Dr. Sunitha Kaiser, MD, MSc, $55016^{\text {th }}$ Street, Box 3214, San Francisco, CA, 94158; Telephone: 415-476-3392; Fax: 415-4765363 E-mail: Sunitha.Kaiser@ucsf.edu

Additional Supporting Information may be found in the online version of this article.
}

Received: January 4, 2018; Revised: March 13, 2018; Accepted: March 15, 2018 ๑) 2018 Society of Hospital Medicine DOI 10.12788/jhm.2988 is important to develop ways to meaningfully measure quality of care and further, to better understand the relationship between measures of quality and healthcare costs.

In December 2016, the National Quality Forum (NQF) endorsed a Pediatric Lower Respiratory Infection (LRI) Readmission Measure. ${ }^{8}$ This measure was developed by the Pediatric Quality Measurement Program, through the Agency for Healthcare Research and Quality. The goal of this program was to "increase the portfolio of evidence-based, consensus pediatric quality measures available to public and private purchasers of children's healthcare services, providers, and consumers."

In anticipation of the national implementation of pediatric readmission measures, we examined whether the Pediatric LRI Readmission Measure could meaningfully identify high and low performers across all types of hospitals admitting children (general hospitals and children's hospitals) using an all-payer claims database. A recent analysis by Nakamura et al. identified high and low performers using this measure ${ }^{10}$ but limited the analysis to hospitals with $>50$ pediatric LRI admissions per year, an approach that excludes many general hospitals. Since general hospitals provide the majority 
of care for children hospitalized with respiratory infections, ${ }^{11}$ we aimed to evaluate the measure in a broadly inclusive analysis that included all hospital types. Because low patient volumes might limit use of the measure, ${ }^{4,6}$ we tested several broadened variations of the measure. We also examined the relationship between hospital performance in pediatric LRI readmissions and healthcare costs.

Our analysis is intended to inform utilizers of pediatric quality metrics and policy makers about the feasibility of using these metrics to publicly report hospital performance and/or identify exceptional hospitals for understanding best practices in pediatric inpatient care. ${ }^{12}$

\section{METHODS}

\section{Study Design and Data Source}

We conducted an observational, retrospective cohort analysis using the 2012-2014 California Office of Statewide Health Planning and Development (OSHPD) nonpublic inpatient and emergency department (ED) databases. ${ }^{13}$ The OSHPD databases are compiled annually through mandatory reporting by all licensed nonfederal hospitals in California. The databases contain demographic (eg, age, gender) and utilization data (eg, charges) and can track readmissions to hospitals other than the index hospital. The databases capture administrative claims from approximately 450 hospitals, composed of 16 million inpatients, ED patients, and ambulatory surgery patients annually. Data quality is monitored through the California OSHPD.

\section{Study Population}

Our study included children aged $\leq 18$ years with LRI, defined using the NQF Pediatric LRI Readmissions Measure: a primary diagnosis of bronchiolitis, influenza, or pneumonia, or a secondary diagnosis of bronchiolitis, influenza, or pneumonia, with a primary diagnosis of asthma, respiratory failure, sepsis, or bacteremia. ${ }^{8}$ International classification of Diseases, $9^{\text {th }}$ edition (ICD-9) diagnostic codes used are in Appendix 1.

Per the NQF measure specifications, ${ }^{8}$ records were excluded if they were from hospitals with $<80 \%$ of records complete with core elements (unique patient identifier, admission date, end-of-service date, and ICD-9 primary diagnosis code). In addition, records were excluded for the following reasons: (1) individual record missing core elements, (2) discharge disposition "death," (3) 30-day follow-up data not available, (4) primary "newborn" or mental health diagnosis, or (5) primary ICD-9 procedure code for a planned procedure or chemotherapy.

Patient characteristics for hospital admissions with and without 30-day readmissions or 30-day ED revisits were summarized. For the continuous variable age, mean and standard deviation for each group were calculated. For categorical variables (sex, race, payer, and number of chronic conditions), numbers and proportions were determined. Univariate tests of comparison were carried out using the Student's $t$ test for age and chi-square tests for all categorical variables. Categories of payer with small values were combined for ease of description (categories combined into "other:" workers' compensation, county indigent programs, other government, other indigent, self-pay, other payer). We identified chronic conditions using the Agency for Healthcare Research and Quality Chronic Condition Indicator $(\mathrm{CCl})$ system, which classifies ICD-9-CM diagnosis codes as chronic or acute and places each code into one of 18 mutually exclusive categories (organ systems, disease categories, or other categories). The case-mix adjustment model incorporates a binary variable for each $\mathrm{CCl}$ category $(0-1,2,3$, or $>4$ chronic conditions) per the NQF measure specifications. ${ }^{8}$ This study was approved by the University of California, San Francisco Institutional Review Board.

\section{Outcomes}

Our primary outcome was the hospital-level rate of 30-day readmission after hospital discharge, consistent with the NQF measure. ${ }^{8}$ We identified outlier hospitals for 30-day readmission rate using the Centers for Medicare and Medicaid Services (CMS) methodology, which defines outlier hospitals as those for whom adjusted readmission rate confidence intervals do not overlap with the overall group mean rate. 5, 14

We also determined the hospital-level average cost per index hospitalization (not including costs of readmissions). Since costs of care often differ substantially from charges, ${ }^{15}$ costs were calculated using cost-to-charge ratios for each hospital (annual total operating expenses/total gross patient revenue, as reported to the OSHPD). ${ }^{16}$ Costs were subdivided into categories representing $\$ 5,000$ increments and a top category of $>\$ 40,000$. Outlier hospitals for costs were defined as those for whom the cost random effect was either greater than the third quartile of the distribution of values by more than 1.5 times the interquartile range or less than the first quartile of the distribution of values by more than 1.5 times the interquartile range. $^{17}$

\section{Analysis}

\section{Primary Analysis}

For our primary analysis of 30-day hospital readmission rates, we used hierarchical logistic regression models with hospitals as random effects, adjusting for patient age, sex, and the presence and number of body systems affected by chronic conditions. ${ }^{8}$ These four patient characteristics were selected by the NOF measure developers "because distributions of these characteristics vary across hospitals, and although they are associated with readmission risk, they are independent of hospital quality of care." ${ }^{10}$

Because the Centers for Medicare and Medicaid Services (CMS) are in the process of selecting pediatric quality measures for meaningful use reporting, ${ }^{18}$ we utilized CMS hospital readmissions methodology to calculate risk-adjusted rates and identify outlier hospitals. The CMS modeling strategy stabilizes performance estimates for low-volume hospitals and avoids penalizing these hospitals for high readmission rates that may be due to chance (random effects logistic model to obtain best linear unbiased predictions). This is particularly important in pediatrics, given the low pediatric volumes in many hospitals admitting children. ${ }^{4,19}$ We then identified outlier hos- 
TABLE 1. 30-day Readmission Rates, Revisit Rates, and Performance Outliers in California Hospitals

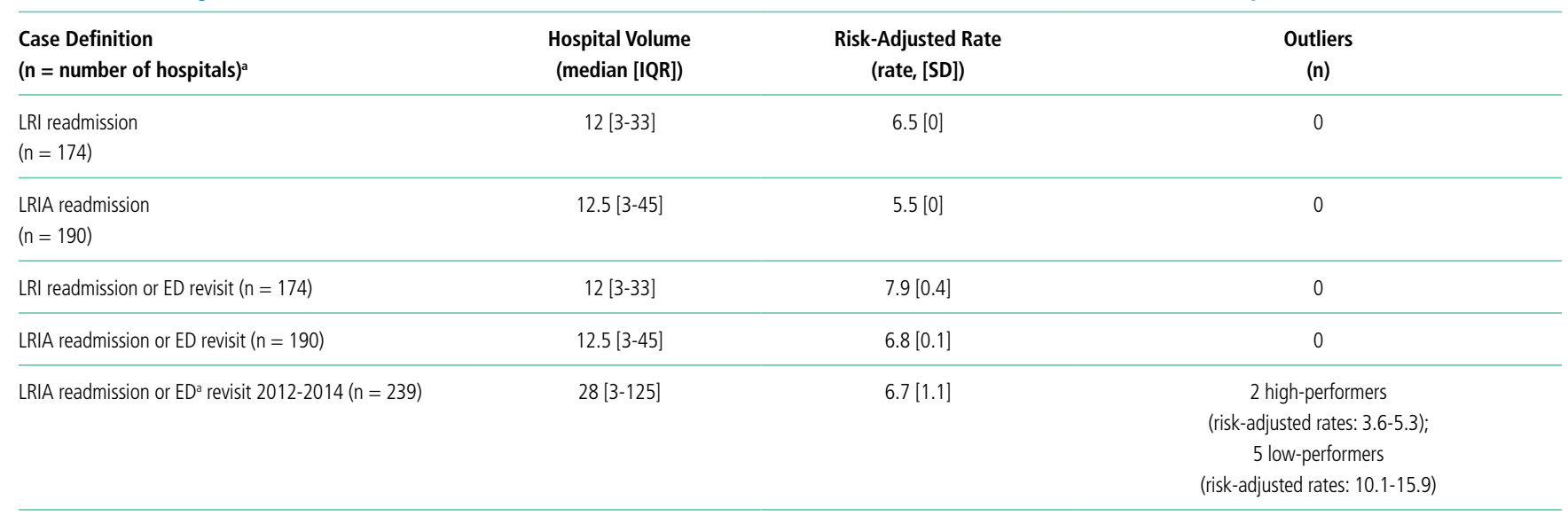

aAll analyses done using 2014 data except for the final analysis, which combined 2012-2014 data.

Abbreviations: ED, emergency department; IQR, interquartile range; LRI, lower respiratory infection; LRIA, lower respiratory infection or asthma; SD, standard deviation.

pitals for the 30-day readmission rate using CMS methodology (hospital's adjusted readmission rate confidence interval does not overlap the overall group mean rate). ${ }^{5,4}$ CMS uses this approach for public reporting on HospitalCompare..$^{20}$

\section{Sensitivity Analyses}

We tested several broadening variations of the NQF measure: (1) addition of children admitted with a primary diagnosis of asthma (without requiring $L R I$ as a secondary diagnosis) or a secondary diagnosis of asthma exacerbation (LRIA), (2) inclusion of 30-day ED revisits as an outcome, and (3) merging of 3 years of data. These analyses were all performed using the same modeling strategy as in our primary analysis.

\section{Secondary Outcome Analyses}

Our analysis of hospital costs used costs for index admissions over 3 years (2012-2014) and included admissions for asthma. We used hierarchical regression models with hospitals as random effects, adjusting for age, gender, and the presence and number of chronic conditions. The distribution of cost values was highly skewed, so ordinal models were selected after several other modeling approaches failed (log transformation linear model, gamma model, Poisson model, zero-truncated Poisson model).

The relationship between hospital-level costs and hospital-level 30-day readmission or ED revisit rates was analyzed using Spearman's rank correlation coefficient. Statistical analysis was performed using SAS version 9.4 software (SAS Institute; Cary, North Carolina).

\section{RESULTS}

Primary Analysis of 30-day Readmissions (per National Quality Forum Measure)

Our analysis of the 2014 OSHPD database using the specifications of the NQF Pediatric LRI Readmission Measure included a total of 5,550 hospitalizations from 174 hospitals, with a mean of 12 eligible hospitalizations per hospital. The mean risk-ad- justed readmission rate was $6.5 \%$ (362 readmissions). There were no hospitals that were considered outliers based on the risk-adjusted readmission rates (Table 1).

\section{Sensitivity Analyses (Broadening Definitions of National Quality Forum Measure)}

We report our testing of the broadened variations of the NQF measure in Table 1. Broadening the population to include children with asthma as a primary diagnosis and children with asthma exacerbations as a secondary diagnosis (LRIA) increased the size of our analysis to 8,402 hospitalizations from 190 hospitals. The mean risk-adjusted readmission rate was $5.5 \%$, and no outlier hospitals were identified.

Using the same inclusion criteria of the NOF measure but including 30-day ED revisits as an outcome, we analyzed a total of 5,500 hospitalizations from 174 hospitals. The mean risk-adjusted event rate was higher at $7.9 \%$, but there were still no outlier hospitals identified.

Using the broadened population definition (LRIA) and including 30-day ED revisits as an outcome, we analyzed a total of 8,402 hospitalizations from 190 hospitals. The mean risk-adjusted event rate was $6.8 \%$, but there were still no outlier hospitals identified.

In our final iteration, we merged three years of hospital data (2012-2014) using the broader population definition (LRIA) and including 30-day ED revisits as an outcome. This resulted in 27,873 admissions from 239 hospitals for this analysis, with a mean of 28 eligible hospitalizations per hospital. The mean risk-adjusted event rate was $6.7 \%$, and this approach identified 2 high-performing (risk-adjusted rates: 3.6-5.3) and 7 low-performing hospitals (risk-adjusted rates: 10.1-15.9).

Table 2 presents the demographics of children included in this analysis. Children who had readmissions/revisits were younger, more likely to be white, less likely to have private insurance, and more likely to have a greater number of chronic conditions compared to children without readmissions/ revisits. 
TABLE 2. Study Population for Broadest Measure Definition

\begin{tabular}{|c|c|c|c|}
\hline & $\begin{array}{l}\text { Children With 30-Day Readmissions } \\
\text { or 30-day ED Revisits } \\
(n=1,832)\end{array}$ & $\begin{array}{c}\text { Children Without 30-Day Readmissions } \\
\text { or 30-Day ED Revisits } \\
(n=26,041)\end{array}$ & $P$ Value \\
\hline \multicolumn{4}{|l|}{ Sex } \\
\hline Male, $n(\%)$ & $1,098(59.9)$ & $15,454(59.3)$ & $.62^{b}$ \\
\hline White & $972(53.1)$ & $13,221(50.7)$ & \\
\hline Black & $273(14.9)$ & $4,162(16.0)$ & \\
\hline Native American/Eskimo/Aleut & $6(0.3)$ & $99(0.4)$ & \\
\hline Asian/Pacific Islander & $114(6.2)$ & $1,861(7.2)$ & \\
\hline Other/Unknown & $461(25.2)$ & $6,461(24.8)$ & \\
\hline Private & $376(20.5)$ & $5,918(22.7)$ & \\
\hline Other & $169(9.2)$ & $1,703(6.5)$ & \\
\hline Number of Chronic Conditions, n (\%) & & & $<.001^{\mathrm{b}}$ \\
\hline $0-1$ & $986(53.8)$ & $19,413(75.6)$ & \\
\hline 2 & $257(14.0)$ & $3,243(12.5)$ & \\
\hline 3 & $185(10.1)$ & $1,420(5.5)$ & \\
\hline$>4$ & $404(20.1)$ & $1,965(7.6)$ & \\
\hline
\end{tabular}

${ }^{a}$ Student's $t$ test; ${ }^{b}$ Chi-square test

Abbreviations: ED, emergency department; SD, standard deviation.

\section{Secondary Outcome: Hospital Costs}

In the analysis of hospital-level costs, we found only one outlier high-cost hospital. There was a $20 \%$ probability of a hospital respiratory admission costing $\geq \$ 40,000$ at this hospital. We found no overall relationship between hospital 30-day respiratory readmission rate and hospital costs (Figure 1). However, the hospitals that were outliers for low readmission rates also had low probabilities of excessive hospital costs (3\% probability of costs $>\$ 40,000$; Figure 2).

\section{DISCUSSION}

We used a nationally endorsed pediatric quality measure to evaluate hospital performance, defined as 30-day readmission rates for children with respiratory illness. We examined all-payer data from California, which is the most populous state in the country and home to one in eight American children. In this large California dataset, we were unable to identify meaningful variation in hospital performance due to low hospital volumes and event rates. However, when we broadened the measure definition, we were able to identify performance variation. Our findings underscore the importance of testing and potentially modifying existing quality measures in order to more accurately capture the quality of care delivered at hospitals with lower volumes of pediatric patients. ${ }^{21}$

Prior analyse have raised similar concerns about the limitations of assessing condition-specific readmissions measures in inpatient pediatrics. Bardach et al. used six statewide databases to examine hospital rates of readmissions and ED revisits for common pediatric diagnoses. They identified few hospitals as high or low performers due to low hospital volumes. ${ }^{5}$ More recently, Nakamura et al. analyzed hospital performance using the same NQF Pediatric LRI Readmission Measure we evaluated. They used the Medicaid Analytic eXtract dataset from 26 states. They identified seven outlier hospitals (of 338), but only when restricting their analysis to hospitals with $>50$ LRI admissions per year. ${ }^{10}$ Of note, if our assessment using this quality measure was limited to only those California hospitals with $>50$ pediatric LRI admissions/year, 83\% of California hospitals would have been excluded from performance assessment.

Our underlying assumption, in light of these prior studies, was that increasing the eligible sample in each hospital by combining respiratory diseases and by using an all-payer claims database rather than a Medicaid-only database would increase the number of detectable outlier hospitals. However, we found that these approaches did not ameliorate the limitations of small volumes. Only through aggregating data over three years was it possible to identify any outliers, and this approach identified only $3 \%$ of hospitals as outliers. Hence, our analysis reinforces concerns raised by several prior analyses ${ }^{4-7}$ regarding the limited ability of current pediatric readmission measures to detect meaningful, actionable differences in performance across all types of hospitals (including general/non- 


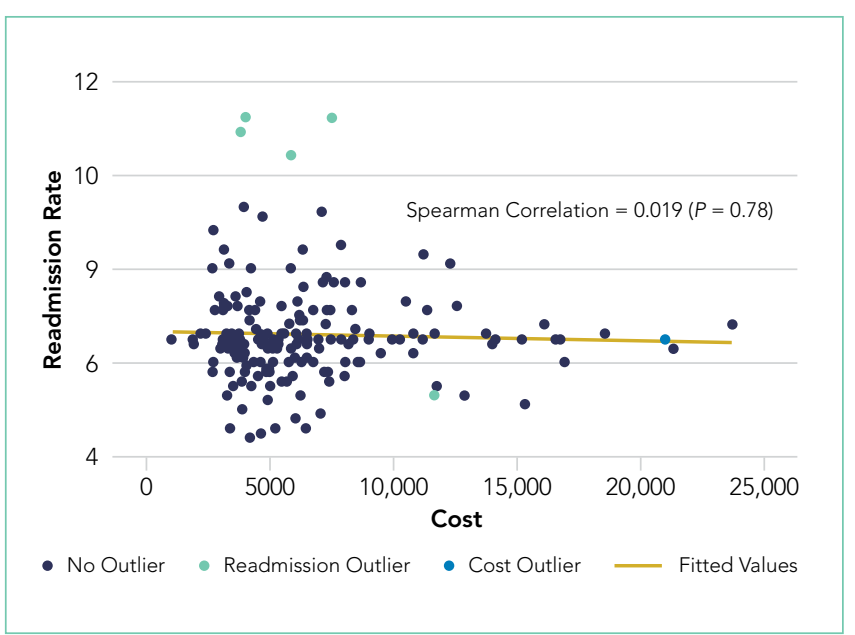

FIG 1. Scatter Plot of Hospital Performance on Readmission/Revisit Rates and Costs. No significant correlation was found between hospital-level 30-day readmission rates for lower respiratory infection and hospital-level average costs for index admissions.

children's hospitals). This issue is of particular concern for common pediatric conditions like respiratory illnesses, for which $>70 \%$ of hospitalizations occur in general hospitals. ${ }^{11}$

Developers and utilizers of pediatric quality metrics should consider strategies for identifying meaningful, actionable variation in pediatric quality of care at general hospitals. These strategies might include our approach of combining several years of hospital data in order to reach adequate volumes for measuring performance. The potential downside to this approach is performance lag-specifically, hospitals implementing quality improvement readmissions programs may not see changes in their performance for a year or two on a measure aggregating three years of data. Alternatively, it is possible that the measure might be used more appropriately across a larger group of hospitals, either to assess performance for a multihospital accountable care organization (ACO), or to assess performance for a service area or county. An aggregated group of hospitals would increase the eligible patient volume and, if there is an ACO relationship established, coordinated interventions could be implemented across the hospitals.

We examined the 30-day readmission rate because it is the current standard used by CMS and all NQF-endorsed readmission measures ${ }^{22,23}$ Another potential approach is to analyze the seven- or 15-day readmission rate. However, these rates may be similarly limited in identifying hospital performance due to low volumes and event rates. An analysis by Wallace et al. of preventable readmissions to a tertiary children's hospital found that, while many occurred within seven days or 15 days, $27 \%$ occurred after seven days and $22 \%$, after $15 .{ }^{24}$ However, an analysis of several adult 30-day readmission measures used by CMS found that the contribution of hospital-level quality to the readmission rate (measured by intracluster correlation coefficient) reached a nadir at seven days, which suggests that most readmissions after the seventh day postdischarge were explained by community- and household-level factors beyond hospitals' control. ${ }^{22}$ Hence, though 7- or 15-day readmission

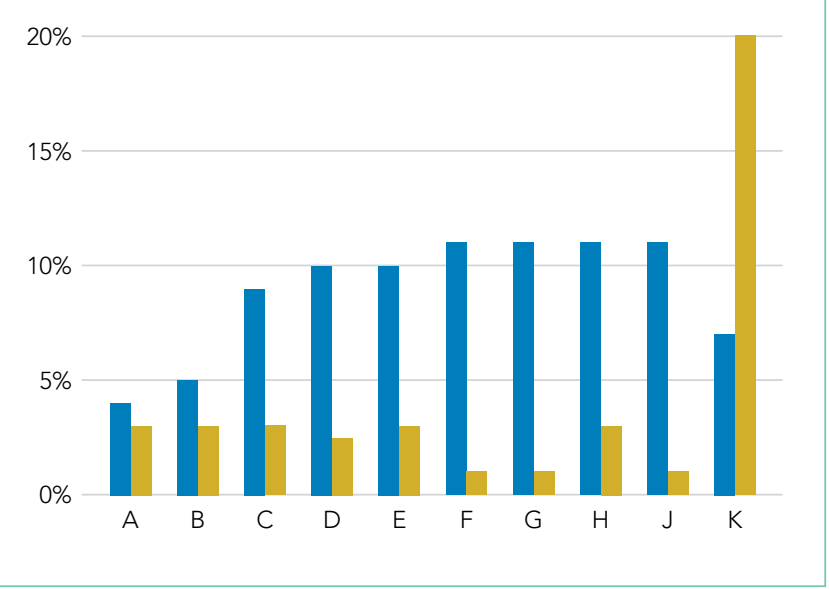

FIG 2. Readmission Rates and Probabilities of High Costs at Outlier Hospitals. Blue bars represent risk-adjusted readmission/revisit rates, and gold bars represent probability of high costs $(>\$ 40,000)$. Hospital A and B had low values for both $(<5 \%)$

rates may better represent preventable outcomes under the hospital's control, the lower event rates and low hospital volumes likely similarly limit the feasibility of their use for performance measurement.

Pediatric quality measures are additionally intended to drive improvements in the value of pediatric care, defined as quality relative to costs. ${ }^{25}$ In order to better understand the relationship of hospital performance across both the domains of readmissions (quality) and costs, we examined hospital-level costs for care of pediatric respiratory illnesses. We found no overall relationship between hospital readmission rates and costs; however, we found two hospitals in California that had significantly lower readmission rates as well as low costs. Close examination of hospitals such as these, which demonstrate exceptional performance in quality and costs, may promote the discovery and dissemination of strategies to improve the value of pediatric care. ${ }^{12}$

Our study had several limitations. First, the OSHPD database lacked detailed clinical variables to correct for additional casemix differences between hospitals. However, we used the approach of case-mix adjustment outlined by an NQF-endorsed national quality metric. ${ }^{8}$ Secondly, since our data were limited to a single state, analyses of other databases may have yielded different results. However, prior analyses using other multistate databases reported similar limitations, ${ }^{5,6}$ likely due to the limitations of patient volume that are generalizable to settings outside of California. In addition, our cost analysis was performed using cost-to-charge ratios that represent total annual expenses/revenue for the whole hospital. ${ }^{16}$ These ratios may not be reflective of the specific services provided for children in our analysis; however, service-specific costs were not available, and cost-to-charge ratios are commonly used to report costs.

\section{CONCLUSION}

The ability of a nationally-endorsed pediatric respiratory readmissions measure to meaningfully identify variation in hospital 
performance is limited. General hospitals, which provide the majority of pediatric care for common conditions such as LRI, likely cannot be accurately evaluated using national pediatric quality metrics as they are currently designed. Modifying measures in order to increase hospital-level pediatric patient volumes may facilitate more meaningful evaluation of the quality of pediatric care in general hospitals and identification of exceptional hospitals for understanding best practices in pediatric inpatient care.

Disclosures: Regina Lam consulted for Proximity Health doing market research during the course of developing this manuscript, but this work did not involve any content related to quality metrics, and this entity did not play any role in the development of this manuscript. The remaining authors have no conflicts of interest relevant to this article to disclose.

Funding: Supported by the Agency for Healthcare Research and Quality (K08 HS24592 to SVK and U18HS25297 to MDC and NSB) and the National Institute of Child Health and Human Development (K23HD065836 to NSB). The funding agency played no role in the study design; the collection, analysis, and interpretation of data; the writing of the report; or the decision to submit the manuscript for publication.

\section{References}

1. Agency for Healthcare Research and Quality. Overview of hospital stays for children in the United States. https://www.hcup-us.ahrq.gov/reports/ statbriefs/sb187-Hospital-Stays-Children-2012.jsp. Accessed September 1, 2017

2. Mendelson A, Kondo K, Damberg C, et al. The effects of pay-for-performance programs on health, health care use, and processes of care: A systematic review. Ann Intern Med. 2017;166(5):341-353. doi: 10.7326/M16-1881.

3. Zuckerman RB, Sheingold SH, Orav EJ, Ruhter J, Epstein AM. Readmissions, observation, and the hospital readmissions reduction program. $N$ Engl J Med. 2016:374(16):1543-1551. doi: 10.1056/NEJMsa1513024.

4. Bardach NS, Chien AT, Dudley RA. Small numbers limit the use of the inpatient pediatric quality indicators for hospital comparison. Acad Pediatr. 2010;10(4):266-273. doi: 10.1016/j.acap.2010.04.025.

5. Bardach NS, Vittinghoff E, Asteria-Peñaloza R, et al. Measuring hospital quality using pediatric readmission and revisit rates. Pediatrics. 2013;132(3):429436. doi: 10.1542/peds.2012-3527.

6. Berry JG, Zaslavsky AM, Toomey SL, et al. Recognizing differences in hospital quality performance for pediatric inpatient care. Pediatrics. 2015;136(2):251 262. doi: 10.1542/peds.2014-3131.

7. Hain PD, Gay JC, Berutti TW, Whitney GM, Wang W, Saville BR. Preventability of early readmissions at a children's hospital. Pediatrics. 2013;131(1):e171-e181. doi: 10.1542/peds.2012-0820.
8. Agency for Healthcare Research and Quality. Pediatric lower respiratory infection readmission measure. https://www.ahrq.gov/sites/default/files/wysiwyg/policymakers/chipra/factsheets/chipra_1415-p008-2-ef.pdf. Accessed September 3, 2017.

9. Agency for Healthcare Research and Quality. CHIPRA Pediatric Quality Measures Program. https://archive.ahrq.gov/policymakers/chipra/pqmpback. html. Accessed October 10, 2017.

10. Nakamura MM, Zaslavsky AM, Toomey SL, et al. Pediatric readmissions After hospitalizations for lower respiratory infections. Pediatrics. 2017;140(2). doi: 10.1542/peds.2016-0938.

11. Leyenaar JK, Ralston SL, Shieh MS, Pekow PS, Mangione-Smith R, Lindenauer PK. Epidemiology of pediatric hospitalizations at general hospitals and freestanding children's hospitals in the United States. J Hosp Med. 2016;11(11):743-749. doi: 10.1002/jhm.2624.

12. Bradley EH, Curry LA, Ramanadhan S, Rowe L, Nembhard IM, Krumholz HM. Research in action: using positive deviance to improve quality of health care. Implement Sci. 2009;4:25. doi: 10.1186/1748-5908-4-25

13. California Office of Statewide Health Planning and Development. Data and reports. https://www.oshpd.ca.gov/HID/. Accessed September 3, 2017.

14. QualityNet. Measure methodology reports. https://www.qualitynet.org/ $\mathrm{dcs} /$ ContentServer?c=Page\&pagename $=$ QnetPublic\%2FPage\%2FOnetTier4\&cid=1219069855841. Accessed October 10, 2017.

15. Riley GF. Administrative and claims records as sources of health care cost data. Med Care. 2009;47(7 Suppl 1):S51-S55. doi: 10.1097/MLR.0b013e31819c95aa.

16. California Office of Statewide Health Planning and Development. Annual financial data. https://www.oshpd.ca.gov/HID/Hospital-Financial.asp. Accessed September 3, 2017.

17. Tukey J. Exploratory Data Analysis: Pearson; London, United Kingdom. 1977.

18. Centers for Medicare and Medicaid Services. Core measures. https://www. cms.gov/Medicare/Quality-Initiatives-Patient-Assessment-Instruments/ QualityMeasures/Core-Measures.html. Accessed September 1, 2017.

19. Berry JG, Toomey SL, Zaslavsky AM, et al. Pediatric readmission prevalence and variability across hospitals. JAMA. 2013;309(4):372-380. doi: 10.1001/ jama.2012.188351

20. Centers for Medicare and Medicaid Services. HospitalCompare. https:// www.medicare.gov/hospitalcompare/search.html. Accessed on October 10, 2017.

21. Mangione-Smith R. The challenges of addressing pediatric quality measurement gaps. Pediatrics. 2017;139(4). doi: 10.1542/peds.2017-0174.

22. Chin DL, Bang H, Manickam RN, Romano PS. Rethinking thirty-day hospital readmissions: shorter intervals might be better indicators of quality of care. Health Aff (Millwood). 2016;35(10):1867-1875. doi: 10.1377/ hlthaff.2016.0205.

23. National Quality Forum. Measures, reports, and tools. http://www.qualityforum.org/Measures_Reports_Tools.aspx. Accessed March 1, 2018.

24. Wallace SS, Keller SL, Falco CN, et al. An examination of physician-, caregiver-, and disease-related factors associated With readmission From a pediatric hospital medicine service. Hosp Pediatr. 2015;5(11):566-573. doi: 10.1542/ hpeds.2015-0015.

25. Porter ME. What is value in health care? N Engl J Med. 2010;363(26):24772481. doi: 10.1056/NEJMp1011024. 